\title{
Capillarity Induced Negative Pressure of Water Plugs in Nanochannels
}

\author{
Niels R. Tas, ${ }^{*, \dagger}$ Petra Mela, ${ }^{\dagger}$ Tobias Kramer, ${ }^{\ddagger}$ J. W. Berenschot, ${ }^{\dagger}$ and \\ Albert van den Berg ${ }^{\dagger}$ \\ MESA+ Research Insitute, University of Twente, P. O. Box 217, \\ 7500 AE Enschede, The Netherlands, and Microsystems Laboratory, \\ Ecole Polytechnique Fédérale de Lausanne, CH-1015 Lausanne, Switzerland
}

Received August 19, 2003

\begin{abstract}
We have found evidence that water plugs in hydrophilic nanochannels can be at significant negative pressure due to tensile capillary forces. The negative pressure of water plugs in nanochannels induces bending of the thin channel capping layer, which results in a visible curvature of the liquid meniscus. From a detailed analysis of the meniscus curvature, the amount of bending of the channel capping can be calculated and used to determine the negative pressure of the liquid. For water plugs in silicon oxide nanochannels of $108 \mathrm{~nm}$ height, a negative pressure of $17 \pm 10$ bar was found. The absence of cavitation at such large negative pressures is explained by the fact that the critical radius for seeding cavities (bubbles) is comparable to the channel height. Scaling analysis of capillarity induced negative pressure shows that absence of cavitation is expected at other channel heights as well.
\end{abstract}

Nanofluidics, the study of fluid behavior in nanoconfinement, is still in its infancy. An important aspect of nanofluidics is the extremely large surface-to-volume ratios, leading to the prominence of surface forces. A clear example is the filling of nanochannels by capillarity, where the wetting properties of channel walls play a crucial role. Dujardin et al. studied this for carbon nanotubes and showed that they can be filled by low surface tension substances such as liquid sulfur, selenium, and cesium. ${ }^{1}$ On a slightly larger scale, capillarity was studied by Sobolev et al., ${ }^{2}$ who measured the capillary pressure of water in quartz capillaries with radii ranging from 200 to $40 \mathrm{~nm}$. Their results indicate that the Young-Laplace equation is valid on $100 \mathrm{~nm}$ length scale, and that on this scale the surface tension of water is equal to its macroscopic value. Silicon micromachining techniques can be used to create nanochannels, which, due to the composition of the channel walls (silicon oxide, silicon nitride), are hydrophilic in nature. ${ }^{3-8}$ We use these nanochannels to study capillarity and use surface tension effects to manipulate aqueous solutions on a picoliter scale. ${ }^{9}$ In our study of capillarity, we observed a peculiar shape of the meniscus of water plugs in nanochannels (Figure 1), which we attribute to a downward bending of the (thin) channel capping under influence of the capillary forces (Figure 2).

To obtain these water plugs, hydrophilic silicon oxide nanochannels ${ }^{10}$ with an approximate height of $100 \mathrm{~nm}$

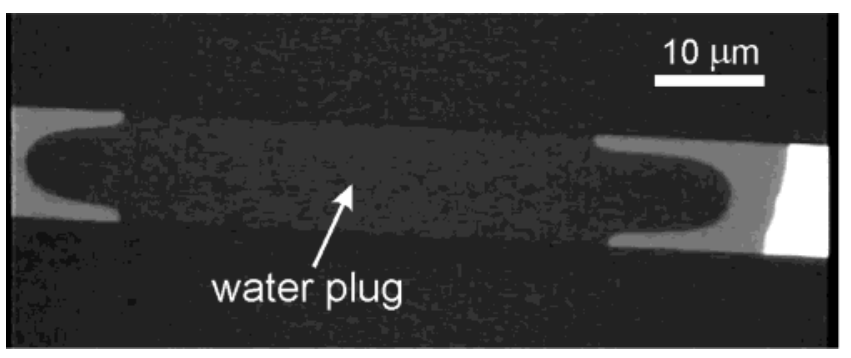

Figure 1. Optical micrograph of a water plug in a $10 \mu \mathrm{m}$ wide nanochannel during drying (top view). The channel height is approximately $100 \mathrm{~nm}$. Note the remarkable shape of the menisci. The channel ends on the right, where a remnant of the sacrificial polysilicon can just be seen. Water vapor exits on the left, where the channel is open.

(Figure 3) were filled with water, and the excess water was removed from the channel entrance. ${ }^{11}$ Subsequently, the water plugs remaining in the channels during drying were observed. We have made a detailed analysis of the meniscus curvature, to estimate the pressure of the water plug in Figure 1. Interestingly, it shows that the tensile capillary forces are so strong on this scale that the water plugs are at a significant negative pressure.

The analysis is based on application of the YoungLaplace equation, which relates the pressure drop across a liquid meniscus to its curvature:

$$
p_{\mathrm{LV}}=\gamma \cdot\left(\frac{1}{r_{1}}+\frac{1}{r_{2}}\right)
$$

* Corresponding author. E-mail: n.r.tas@el.utwente.nl.

$\doteqdot$ Ecole Polytechnique Fédérale de Lausanne. 


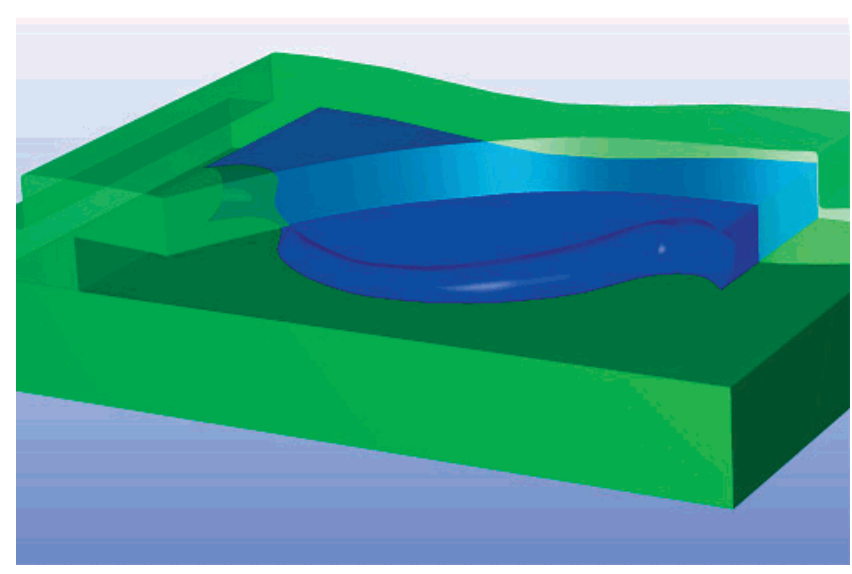

Figure 2. Artist impression of the shape of the meniscus as related to the bending of the channel capping. Part of the capping has been left out to show the meniscus more clearly.

where $p_{\mathrm{LV}}=p_{\mathrm{L}}-p_{\mathrm{V}}$ is the pressure difference between the liquid side (at $p_{\mathrm{L}}$ ) and the vapor side (at $p_{\mathrm{V}}$ ) of the meniscus, $\gamma$ is the surface tension of the liquid, and $r_{1}, r_{2}$ are the radii of curvature of any two normal sections of the meniscus perpendicular to one another. ${ }^{12}$ The radii of curvature are taken negative for a concave curvature of the liquid surface. As the pressure inside as well as outside of the meniscus is uniform along the meniscus, the Laplace pressure should be equal at different positions at the meniscus. For two positions at the meniscus, one at the center of the channel (position $c$, Figure 4a) and one at the edge of the channel (position $e$, Figure $4 a)$. We can therefore write:

$$
\frac{1}{r_{1 c}}+\frac{1}{r_{2 c}}=\frac{1}{r_{1 e}}+\frac{1}{r_{2 e}}
$$

where $r_{\mathrm{lc}, \mathrm{e}}$ are the radii of curvature in the $x y$-plane, and $r_{2 c, e}$ in the $x z$-plane (Figure 4a,b). Simple geometry applied to Figure $4 \mathrm{~b}$ relates the curvature in the $x z$-plane to the channel heights $h_{\mathrm{c}}$ and $h_{\mathrm{e}}$ at positions $c$ and $e$ :

$$
r_{2 c, e}=\frac{-h_{c, e}}{2 \cos \theta}
$$

Combining eqs 2 and 3 gives an expression for the capping deflection $z=h_{\mathrm{e}}-h_{\mathrm{c}}$ at the center (position $c$ ) of the channel.

$$
z=h_{e}-h_{c}=h_{e}+\frac{2 \cos \theta}{\frac{1}{r_{1 e}}-\frac{2 \cos \theta}{h_{e}}-\frac{1}{r_{1 c}}}
$$

Using the measured radii $r_{1 \mathrm{c}, \mathrm{e}}$, contact angle $\theta$ and initial channel height $h_{\mathrm{e}}$, a center deflection $z=11.0 \pm 2.8 \mathrm{~nm}$ results. ${ }^{13,14}$ This value includes the initial center deflection $z_{\mathrm{i}}=3.5 \pm 3.0 \mathrm{~nm}$ already present in the empty channel, caused by internal stresses in the capping layer. Subtraction of $z_{\mathrm{i}}$ yields the center deflection $z_{\mathrm{p}}=7.5 \pm 4.0 \mathrm{~nm}$ of the channel capping caused by the pressure difference $\Delta p$ across it. ${ }^{14}$ Elastic mechanics can be used to relate $\Delta p$ with the center deflection $z_{\mathrm{p}}$ :

$$
\Delta p=p_{0}-p \cong \frac{64 \cdot E \cdot t^{3} \cdot z_{\mathrm{p}}}{w^{4}}
$$

where $E=70 \pm 7 \mathrm{GPa}$ is the Young's modulus of the capping layer, $w=10.2 \pm 0.3 \mu \mathrm{m}$ is the width of the channel, and $t=832 \pm 40 \mathrm{~nm}$ is the thickness of the capping layer. Equation 5 produces a pressure difference $\Delta p=18$ \pm 10 bar, ${ }^{14}$ which implies that there exists a significant negative pressure $p=-17 \pm 10 \mathrm{bar}$ in the water plug.

A simple model for the capillary action can be used for a theoretical prediction of the negative pressure. The tensile capillary forces $F_{\text {cap }}$ (Figure 5) are found from the change in the surface free energy by wetting of the channel walls. They are counterbalanced by the pressure drop across the liquid meniscus (the capillary pressure $p_{\text {cap }}$ ), which for a flat hydrophilic channel of rectangular cross section is given by: ${ }^{9}$

$$
p_{\text {cap }}=\frac{2 \gamma \cos \theta}{h}
$$

where $\gamma$ is the surface tension of the liquid, $h$ is the channel height, and $\theta$ is the contact angle of the meniscus with the channel walls. For water at room temperature, a channel height of $108 \mathrm{~nm}$ and a contact angle of $18^{\circ}$, eq 6 predicts a capillary pressure of 13 bar. For an ambient pressure $p_{0}=$ 1 bar this implies that the water plug is at a pressure of -12 bar. This compares well with the measured value, taking into account its error margin.

The experimentally obtained negative pressure is large, but is well below the theoretical tensile strength of water, which is estimated between 500 and 3000 bar. $^{15}$ The presented method is a new and relatively easy way to induce negative pressure in liquid, as compared to the traditional isochoric cooling method ${ }^{16,17}$ or the centrifugal method. ${ }^{18}$ The large negative pressure in the water plug (at least 7 bar) raises the question why cavitation of the water does not occur. Liquid at a pressure below the vapor pressure is in a metastable state. ${ }^{19}$ Small gas bubbles may act as seeding points for cavitation. There is a critical radius of cavities, ${ }^{20,21}$ beyond which they grow virtually unlimited: $R_{\text {crit }}=2 \gamma /-p$, where $p$ is the (negative) pressure of the liquid surrounding the cavity and the absolute pressure inside of the cavity is neglected. At the limit, the work $-p \mathrm{~d} V$ of the growing cavity equals the work $\gamma \mathrm{d} A$ needed to create the extra area of the meniscus. For $R<R_{\text {crit }}$ the ratio $\mathrm{d} A / \mathrm{d} V=2 / R$ is larger than at the limit, and cavities do not grow. At $p=-17$ bar in water at room temperature, $R_{\text {crit }}=0.9 \times 10^{2} \mathrm{~nm}$. The critical radius is comparable to the height of the channel, and it is therefore likely that the number of bubbles of critical size and larger that enter the channel during filling is small. This might explain why cavitation did not occur, although we did not degass the water and evacuate the channels prior to 


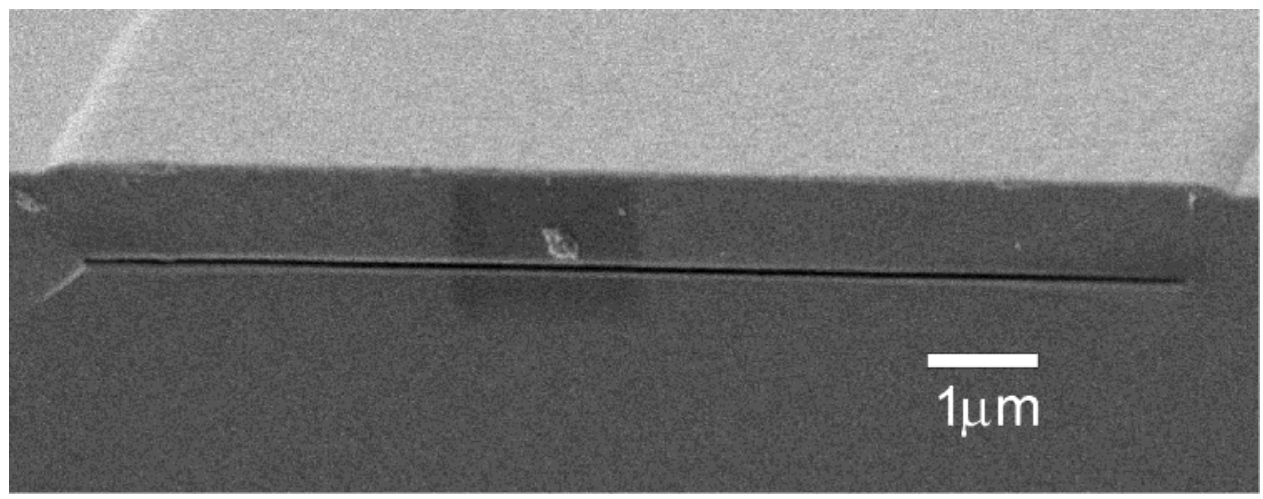

Figure 3. Cross section (SEM) of a nanochannel used in the experiments. The channel height is approximately $100 \mathrm{~nm}$.

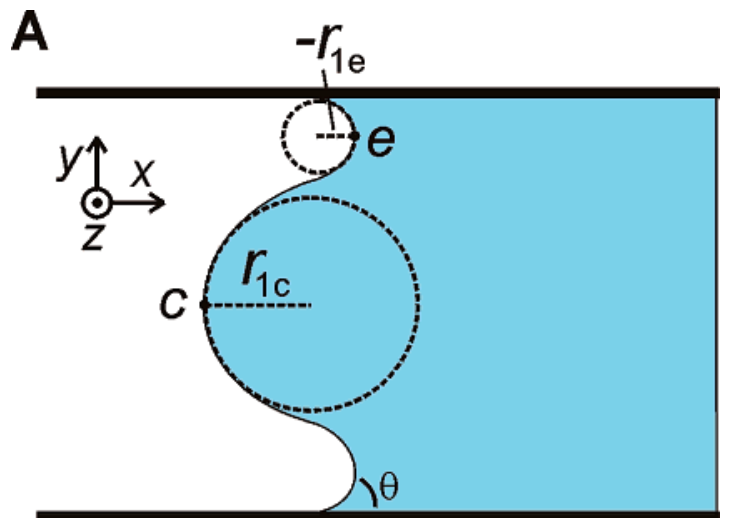

B

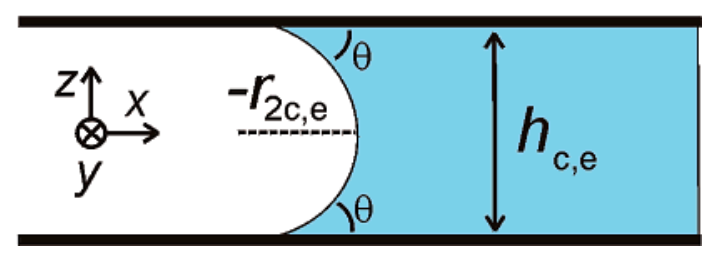

Figure 4. Meniscus curvature at positions $c$ (center) and $e$ (edge). (A) In the $x y$-plane, top view of the channel. Here $r_{1 c}$ and $r_{1 e}$ are the radii of curvature, and $\theta$ is the contact angle. (B) In the $x z$ plane, side view of the channel. Here $h_{c}$ and $h_{e}$ are the channel heights and $r_{2 c}$ and $r_{2 e}$ the radii of curvature at positions $c$ and $e$, respectively.

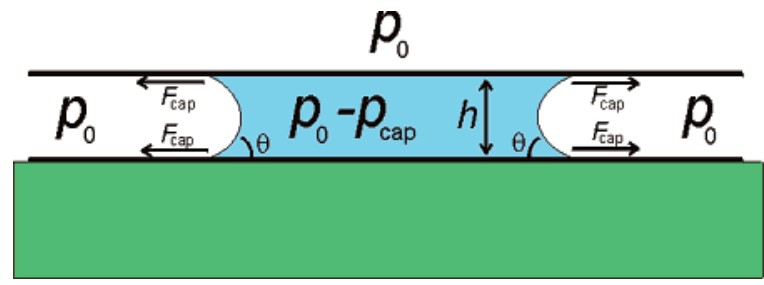

Figure 5. Representation of a water plug in a flat hydrophilic channel (side view), where $h$ is the channel height and $\theta$ is the contact angle. The tensile capillary forces $F_{\text {cap }}$ result in a lowering of the pressure of the water plug by $p_{\text {cap }}$, the capillary pressure.

filling. ${ }^{22}$ Since the capillarity-induced negative pressure $p$ scales with $1 / h$ and the critical radius scales with $1 / p$, the critical radius scales proportional to $h$, so the absence of cavitation is expected also for other channel heights. This means that the developed method may offer a unique opportunity to induce even larger negative pressures without the risk of the occurrence of cavitation.

The observed effect, liquid at negative pressure due to tensile capillary forces, is of general importance for understanding the behavior of liquid in nanopores and cracks. Capillarity in submicron gaps is an issue in the field of microstructure fabrication, where microstructure collapse during drying and the consequent permanent adherence ("stiction") is a notorious problem. ${ }^{23-25}$ It will be important for nanotribology, ${ }^{26}$ in order to understand the properties of lubrication films in nanoconfinement.

Acknowledgment. The authors are grateful to S. Onclin, E. de Vries, E. Oosterbroek, and J. van Nieuwkasteele for their technical assistance. The stimulating discussions with D. Lohse, S. Hilgenfeldt, J. Eijkel, H. Jansen, and M. Elwenspoek are greatly appreciated.

\section{References}

(1) Dujardin, E.; Ebbesen, T. W.; Hiura, H.; Tanigaki, K. Science 1994 265,1850 .

(2) Sobolev, V. D.; Churaev, N. V.; Velarde, M. G.; Zorin, Z. M. J. Colloid Interface Sci. 2000, 222, 51.

(3) Stern, M. G.; Geis, M. W.; Curtin, J. E. J. Vac. Sci. Technol. 1997 B15, 2887.

(4) Han, J.; Craighead, H. G. Science 2000, 288, 1026.

(5) Foquet, M.; Korlach, J.; Zipfel, W.; Webb, W. W.; Craighead, H. G. Anal. Chem. 2002, 74, 1415.

(6) Cao, H. et al. Appl. Phys. Lett. 2002, 81, 174.

(7) Tas, N. R.; Berenschot, J. W.; Mela, P.; Jansen, H. V.; Elwenspoek, M; van den Berg, A. Nano Lett. 2002, 2, 1031

(8) Haneveld, J.; Jansen, H. V.; Berenschot, J. W.; Tas, N. R.; Elwenspoek, M. J. Micromech. Microeng. 2003, 13, S62.

(9) Tas, N. R.; Berenschot, J. W.; Lammerink, T. S. J.; Elwenspoek, M.; van den Berg, A. Anal. Chem. 2002, 74, 2224.

(10) Nanochannels were fabricated by the sacrificial layer method on a fused silica substrate wafer, using a tetra-ethyl-ortho-silicate (TEOS) silicon oxide layer as the channel capping. Channels with a length of approximately $375 \mu \mathrm{m}$ were etched by dissolving a polysilicon sacrificial layer in a 5\% tetra-methyl-ammonium-hydroxide (TMAH) solution, with additives to increase selectivity and etch speed. Channels were dried by first replacing the etchant by water, subsequently replacing the water by isopropyl alcohol (both replacements were carried out by diffusion, as rinsing is practically difficult in these small channels), and finally evaporating the alcohol during spinning of the wafer. The sacrificial layer method produces slightly tapered channels of approximately $200 \mathrm{~nm}$ height at the entrance down to $86 \mathrm{~nm}$, the initial polysilicon thickness, at the channel end.

(11) To fill the channel, a droplet of deionized water $(0.08 \mu \mathrm{S} / \mathrm{cm})$ was deposited on the channel entrance by careful handling using a syringe with a needle (Microlance $0,3 \times 13 \mathrm{~mm}$ ). After filling of the channel, the water was removed from the entrance by capillary suction of a 
tissue. After this, the water in the channel would evaporate in a few minutes. During the final stage of the drying process, the remaining water plug with the peculiarly shaped menisci stayed in the most narrow part of the slightly tapered channel.

(12) Hunter, R. J. Foundations of Colloid Science, 2nd ed.; Oxford University Press: Oxford, 2001; pp 53-55.

(13) Strictly spoken, to obtain the radii of curvature $r_{1 c, e}$, the intersection of the $x y$-plane with the meniscus should be taken at half the channel height, because then it is a normal section. However, due to the small radius of curvature $r_{2}$ in the $x z$-plane, the curvature visible in the top view, Figure 1, is representative of the curvature at half the channel height, and was used for the measurement. We focus our analysis on the left meniscus of Figure 1, as the situation at the right meniscus is more complicated due to the unknown pressure of the trapped air that was compressed during filling. Following the definition of Figure $4 \mathrm{a}$, the values of $r_{1 c, e}$ have been measured for the left meniscus of Figure 1: $r_{1 c}=2.66 \pm 0.21 \mu \mathrm{m}$ and $r_{1 e}=-0.61 \pm 0.13 \mu \mathrm{m}$. The contact angle of water on silicon dioxide was measured using a Krüss G 10 contact angle measuring system equipped with a CCD camera. The advancing and receding contact angles were determined on top of the silicon dioxide capping layer during the respective increase and decrease of the volume of a water drop. The meniscus was observed during drying. For the contact angle we therefore apply the measured value of the receding contact angle, $\theta=18 \pm 8^{\circ}$. The initial centre deflection of the channel capping and the channel height were measured using a Micromap 512 optical interference surface profiler, at $632 \mathrm{~nm}$ wavelength and with a $40 \times$ objective. Position $e$ is close to the channel edge. Therefore, it is assumed that the channe height $h_{\mathrm{e}}$ is equal to the initial channel height, which at the position of the left meniscus of Figure 1, has a value of $108 \pm 11 \mathrm{~nm}$.
(14) Error propagation was calculated using a statistical approach. For a derived variable $f$, which is a function of measured variables $x_{\mathrm{i}}$ with random and independent errors $\Delta x_{i}$, the error is given by $\sqrt{ }(\Sigma(\partial f)$ $\left.\left.\left.\partial x_{\mathrm{i}}\right)^{2} \cdot \Delta x_{\mathrm{i}}^{2}\right)\right)$. The error margins we used in the calculations are typically the $3 \sigma$ values. To determine the mean value and the standard deviation $\sigma$ we performed repeated measurements of radii of curvature, channel height, and initial center deflection. The error margins for the Young's modulus, capping layer thickness, and contact angle were estimated.

(15) Trevena D. H. Cavitation and tension in liquids; Adam Hilger: Bristol, 1987; p 15.

(16) Zheng, Q.; Durben, D. J.; Wolf, G. H.; Angell, C. A. Science 1991, $254,829$.

(17) Henderson, S. J.; Speedy, R. J. J. Phys. E: Sci. Instrum. 1980, 13, 778.

(18) Briggs, L. J. J. Appl. Phys. 1950, 21, 721.

(19) Speedy, R. J. J. Phys. Chem. 1982, 86, 982.

(20) Goedecke, N.; Eijkel, J.; Manz, A. Lab Chip 2002, 2, 219.

(21) Steudle, E. Annu. Rev. Plant Physiol. Plant Mol. Biol. 2001, 52, 847.

(22) Overton, G. D. N.; Edwards, M. J.; Trevena, D. H. J. Phys. D.: Appl. Phys. 1982, 15, L129.

(23) Guckel, H.; Sniegowski, J. J.; Christenson, T. R.; Mohney, S.; Kelly, T. F. Sens. Actuators 1989, 20, 117.

(24) Mastrangelo, C. H.; Hsu, C. H. J. Microelectromech. Syst. 1993, 2(1), 33.

(25) Tas, N. R.; Sonnenberg, A. H.; Jansen, H. V.; Legtenberg, R.; Elwenspoek, M. J. Micromech. Microeng. 1996, 6, 385.

(26) Bhushan, B. Tribology Int. 1995, 28, 85.

NL034676E 